\title{
sciendo
}

\section{A Finite Element Model for Estimation of Contact Dynamics During a Jumping Movement on a Trampoline}

\author{
by \\ Jingguang Qian ${ }^{1}$, Yiling Mao ${ }^{2}$, Xiao Tang ${ }^{1}$, Zhaoxia $\mathrm{Li}^{2}$, Chen Wen ${ }^{3}$, \\ Songning Zhang ${ }^{3}$
}

In order to fully understand contact dynamics on a trampoline, a simulation approach using a musculoskeletal model coupled with a dynamic model of the trampoline is essential. The purpose of the study was to examine dynamics and selected lower extremity muscle forces in a landing and jumping movement on a trampoline, using a combination of finite element modeling and musculoskeletal modeling. The rigid frame of the trampoline was modeled in ADAMS and coupled with a finite element model of the elastic trampoline net surface in ANSYS. A musculoskeletal model of an elite trampoline athlete was further developed in LifeMod and combined with the finite element model of the trampoline. The results showed that the peak trampoline reaction forces (TRF) were $3400 \mathrm{~N}(6.6 \mathrm{BW})$ and $2900 \mathrm{~N}(5.6 \mathrm{BW})$ for the left and right limb, respectively. The right hip, knee and ankle joint reaction forces reached the maximum between 3000-4000 $\mathrm{N}(5.8-7.7 \mathrm{BW})$. The gluteus maximum and quadriceps reached the maximum muscle force of $380 \mathrm{~N}(0.7 \mathrm{BW})$ and 780 $N(1.5 \mathrm{BW})$, respectively. Asymmetric loading patterns between left and right TRFs and lower extremities joint reaction forces were observed due to the need to generate the rotational movement during the takeoff. The observed rigid and erect body posture suggested that the hip and knee extensors played important roles in minimizing energy absorption and maximizing energy generation during the trampoline takeoff.

Key words: trampoline, finite element model, musculoskeletal model, landing technique.

\section{Introduction}

Both recreational and competitive use of trampolines have seen rapid growth around the world in recent years. Trampoline competitions require athletes to perform 10 different somersault movements of various difficulty on a trampoline of $4.28 \times$ (length) $\times 2.14$ (width) $\times 1.5 \mathrm{~m}$ (height) consecutively in a single bout (FIdG, 2015). Performances are judged based on numbers of somersaults, degrees of rotation, and posture. In 2015, Federation of International Gymnastics Technical Regulations (2015) published the new revised scoring methods for gymnastic performance, which redefined the difficulty level of all gymnastic movements to promote innovations. With the increasing performance

difficulties, it is necessary to improve the vertical jump height in order to complete high quality movements. It is, therefore, critical for athletes to utilize the elasticity of trampoline to improve the jump height and therefore, to achieve twisting somersaults with a high degree of difficulty.

Many studies have been performed on trampoline exercises (Ando and Yamamoto, 1987; Arora et al., 2016; Atilgan, 2013; Blajer and Czaplicki, 2001; Burke et al., 2015; Chen et al., 2014; Eager et al., 2012; Khorashad, 2013; Kuisis et al., 2011; Vaughan, 1980). However, various different jumping related movements on a trampoline have not been well examined (Blajer and Czaplicki, 2003). The takeoff from a trampoline is considered

\footnotetext{
1 - Department of Sport Science, Nanjing Sport Institute, Nanjing, China.

2 - Department of Engineering Mechanics, Southeast University, Nanjing, China.

3 - Biomechanics/Sports Medicine Lab, The University of Tennessee, Knoxville, TN, USA
} 
the most important component of trampoline movements, and determines the vertical jump height and successful completion of a whole movement set with high degrees of difficulty (Peng et al., 2013). Jumping movements on a trampoline net are directly related to dynamics of the human movement and its interaction with the net. Traditional biomechanical tools, i.e. motion capture and force measurements, are not sufficient to examine the process of the interaction dynamics between the human body and the trampoline. In order to investigate the interaction between the athlete and the elastic net surface of the trampoline, the simulation of the musculoskeletal model coupled with the dynamic model of the trampoline is needed to comprehensively understand trampoline jumping movements. We developed a biomechanical finite element model (FEM) of a trampoline bed for examination of trampoline gymnastic performance (Qian and Ye, 2016). However, to our knowledge, no studies have integrated an FEM with a human musculoskeletal modeling approach in examining the interaction of a trampoline's elastic net surface and an athlete performing a jumping activity on a trampoline.

Trampoline simulation software, VHTrampoline, was developed to allow modifications and simulation of entire movement sets based on high-speed motion capture data (Wang, 2007). Kinematic characteristics of takeoff movements on a trampoline performed by an elite female athlete were captured and analyzed first and then simulated using LifeMod (Li, 2012). Lv (2012) performed a modeling and simulation study of the trampoline related movements, which included an FEM of the center area of the trampoline in ANASYS, motion capturing of jumping movements, and the trampoline reaction forces applied to the athlete during the jumping movements. Liu (2014) also modeled the trampoline with shell elements in ABAQUS. The force and displacement relationship of the net was established through the measured displacements under applications of known weights at the center location of the net and through curve fitting, which was used to verify the finite element net model. Although these studies investigated the dynamic characteristics of the trampoline and human movement separately, no previous studies have been conducted to integrate the human movement dynamics on a trampoline with a trampoline FEM. The challenge of biomechanical research on trampoline related movements is therefore the simulation of interaction between the trampoline and human movement. Furthermore, it is necessary to use a musculoskeletal modeling approach to investigate muscle contributions to jumping movement on a trampoline. Therefore, the purpose of the study was to examine dynamics and selected lower extremity muscle forces in a landing and jumping movement on a trampoline, using a combination of finite element modeling and musculoskeletal modeling.

\section{Methods}

\section{Modeling of the trampoline}

The entire modeling process of a trampoline and a human was performed in four different steps: 1) conducting static load experiments on a trampoline to obtain the equivalent stiffness of the center of the trampoline mesh surface, 2) conducting a dynamic loading experiment on the trampoline to obtain the effective damping ratio, 3) establishing control functions of the human and trampoline interactions based on the results obtained from the static and dynamic loading experiments, and 4) establishing dynamic modeling of the human body and integrating it with a finite element model of the trampoline net surface. More details about the steps 1-3 can be found elsewhere (Qian and Ye, 2016) and a brief account is provided here. Step 4 and the associated motion capture of the trampoline movement are the focus of this study.

\section{Static experiment}

Both static and dynamic load experiments were performed to examine the relationship between the loads and the vertical displacements of the net central region. The trampoline had a dimension of 5.05 (length) x 2.91 (width) x 1.15 (height) $\mathrm{m}$ and was mainly composed of elastic springs and a fiber net. A series of static loads $\left(F_{y}\right)$ of 20 to $260 \mathrm{~kg}$ with a combination of $20 \mathrm{~kg}$ barbells (Figure 1) was applied to the net center in an area of $552 \mathrm{~cm}^{2}$ and a diameter of $37.5 \mathrm{~cm}$. The center of the trampoline net was set as the origin of the net coordinate system $\mathrm{O}(0,0,0)$, and four points in the net central region were further identified, A (-18.7 $\mathrm{cm}, 0,0), \mathrm{B}(0,-20.55 \mathrm{~cm}, 0), \mathrm{C}(18.9 \mathrm{~cm}, 0,0)$, and D $(0,20.15 \mathrm{~cm}, 0)$, where the barbell loads were applied. Five tape measures were affixed to the floor and used to measure the vertical 
displacements of the five points in the net central region under the various loads, if the average vertical displacements of points $A$ and $C$ and points $\mathrm{B}$ and $\mathrm{D}$ were significantly different from that of the center point $O$.

Through cubic spline fitting, the relationship between $F_{y}$ and $y$ (average vertical displacements of five points, $O, A, B, C$ and $D$ ) was determined as equation 1 .

$$
F_{y}=3.65 y+1.97 y^{2}+4.76 y^{3}
$$

We defined the equivalent stiffness, $k_{3}$, as the ratio of $F_{y}$ and $y$ :

$$
k_{3}=F_{y} / y=3.65+1.97 y+4.76 y^{2}
$$

It is apparent that $k_{3}$ is nonlinear. The dynamic characteristic of the nonlinear dynamic system is very complex. Therefore, in order to facilitate the study of the problem, we simplified the trampoline model. We found that constant term $k_{3}$ dominated within the range of its measurements. The equivalent stiffness of the center of trampoline mesh surface was simplified as linear stiffness.

$$
F_{y}=5.69 y-0.023
$$

Therefore, the equivalent stiffness was determined as $5.69 \mathrm{KN} / \mathrm{m}$.

\section{Dynamic loading experiment}

For the dynamic loading test, a small $5 \mathrm{~kg}$ ball was placed at the net center location, and a downward excitation was then applied to the ball to cause it to be projected vertically upward. The ball's maximum and subsequent vertical displacements were recorded and measured using a high-speed video camera. Through the measured decayed vibration displacements and mass of the ball, the effective damping ratio of the trampoline net bed was determined.

\section{Finite element model}

ADAMS (Automatic Dynamic Analysis of Mechanical System, Mechanical System, CA, USA) is one of most widely used multibody dynamics software packages with the capability to rapidly develop dynamic models. In this study, the human body model was established using LifeMod (LifeModeler, Inc., San Clemente, CA), the biomechanical plug-in to the ADAMS. However, the LifeMod can only define rigid contact models. Since a trampoline bed is made of nylon ropes, a rigid plate with $40 \times 20 \mathrm{~cm}$ was established in the center of the trampoline. The ADAMS is capable of solving elastic structural problems. In order to model the trampoline bed surface precisely, a finite element model (FEM) of the trampoline bed was developed using both the ADAMS and ANSYS (ANSYS, INC., Cecil Township, Pennsylvania, USA).

Since the ADAMS can only be used to construct simple elastic components and the trampoline net is a surface structure that has large elasticity and experiences large deformation during trampoline movements, the usage of specialized finite element modeling software of ANSYS is necessary in developing a finite element model of the trampoline bed. The modal neutral files (MNF), containing the deflection characteristics, location and connectivity of nodes, mass and moment of inertia of nodes, different kinds of modal, stiffness and generalized mass and width of modal, were obtained using ANSYS and imported into the ADAMS as a compensation for the disadvantages of the rigid model developed in the ADAMS. Therefore, the dynamic characteristics of the trampoline bed were more accurately established.

The elastic trampoline bed surface was then imported into the ADAMS. A total of 112 markers were placed and connected to the four edges of the trampoline bed. The elasticity coefficients of 0.7 $\mathrm{KN} / \mathrm{m}$ and $1.10 \mathrm{KN} / \mathrm{m}$ obtained through the static loading experiments were set for the thin and thick spring, respectively. The rigid plate of $40 \mathrm{~cm}$ (length) $\times 20 \mathrm{~cm}$ (width) $\times 0.2 \mathrm{~cm}$ (height) was fixed and set in the center of the trampoline bed model in the ADAMS

In order to verify the accuracy of the modeled trampoline bed compared to the experimental data obtained in the static load tests described above, static loads were applied to the center plate of the trampoline bed model. The plate downward displacements measured from the static load tests $(\delta t)$ and ADAMS simulations ( $\delta a)$ were obtained under the same loading conditions. The relative error was defined as: $\varepsilon=\frac{\delta t-\delta a}{\delta t}$. The results showed that the maximum error was less than $8 \%$ across all loading conditions (Figure 1), suggesting that the surface net model built in ADAMS was valid.

Trampoline Exercise and Motion Capture

The participant for this study (body mass: 58 $\mathrm{kg}$, body height: $1.69 \mathrm{~m}$, age: 23 years) was an athlete in the Chinese National Trampoline 
Championship, Guangyuan Construction Cup, Anji, Zhejiang, China, August 2013. The participant completed a group 811 exercise that requires the athlete to complete a double backward somersault $\left(720^{\circ}\right)$ with a complete twist $\left(360^{\circ}\right)$. Two high-speed digital video camcorders $(100 \mathrm{~Hz}$, Casio EX700, Japan) were set up with optical axes perpendicular to each other and used to capture the sagittal and frontal plane jumping movements of the athlete, respectively (Figure 2). A 3D calibration frame was filmed before the completion (Figure 2). The video data were digitized using the Ariel Performance Analysis System (APAS, Ariel Dynamics Inc. Trabuco Canyon, CA). The digitized marker trajectories were imported to the LifeMod for further analyses. Informed consent was obtained prior to the motion data collection.

Modeling of the Human Body and Trampoline Dynamics

A customized skeleton model was established based on the participant's height, mass, gender, age as well as a series of anthropometric measurements (Figure 3a). A total of 118 muscles was then created at relevant anatomical landmarks (Figure $3 b$ ). Kinematic trajectories from the collected kinematics were then prescribed to the model to drive and control its motion (Figure 3c).

Once the contact between participant's feet and the trampoline was established, the finite element model of the trampoline was combined and coupled with the musculoskeletal model (Figure 3d). With the application of gravity to the model, jumping trajectories were simulated and minimized between the motion capture data and the inverse dynamics simulation results in the Analyze module of LifeMod. Change of the joint and soft tissue during the inverse dynamic simulation was recorded and ready to be used in the forward dynamic simulation. Then, the joints and muscles were trained according to the results of the previous simulation in the Training module. After that, a tracking agent obtained from the inverse dynamic was able to initiate the forward dynamic simulation and created the dynamic model.

\section{Results}

From the motion capture data, the height and related time were found to be $4.706 \mathrm{~m}$ (from ground) and $0.98 \mathrm{~s}$ for the maximum jump height and $0.98 \mathrm{~m}$ and $0.18 \mathrm{~s}$ for the lowest body position, respectively, and the trampoline net height was measured as $1.15 \mathrm{~m}$. The ankle, knee and hip angles were found to be $5.2^{\circ}, 38.9^{\circ}$ and $39.5^{\circ}$ at the foot contact with the trampoline, respectively.

The simulated trampoline reaction forces (TRF) in LifeMod were the external force applied to the athlete. Figure 4 showed the patterns of the TRF during the foot contact on the trampoline. The left and right TRFs showed slightly different timing and patterns. At the lowest point of the trampoline jump, the peak TRF was $3400 \mathrm{~N}(6.6$ body weight (BW)) and $2900 \mathrm{~N}(5.6 \mathrm{BW})$ for the left and right limb, respectively.

The hip, knee and ankle joint reaction forces of the right lower limb are presented in Figure 5a. They increased gradually until reaching their maximum between $3000-4000 \mathrm{~N}$ (5.8 BW - 7.7 $\mathrm{BW})$ at the foot contact with the trampoline, and decreased thereafter. The hip, knee and ankle joint reaction forces of the left lower limb showed different patterns (Figure 5b). The left ankle joint reaction force reached a minimum reaction force of $710 \mathrm{~N}(1.25 \mathrm{BW})$ soon after the foot contact and then increased to its maximum after the foot contact. The left knee and hip joint reaction forces reached their respective maximum prior to the foot contact and then decreased to reach their respective minimum of $1116 \mathrm{~N}(1.96 \mathrm{BW})$ and 1158 $\mathrm{N}(2.0 \mathrm{BW})$ soon after the initial contact, and then increased to about $1600 \mathrm{~N}$ (2.81 BW) after the feet contact.

Both right and left muscle gluteus maximus forces reached their maximum values of about 380 $\mathrm{N}$ at the foot contact, and decreased rapidly to their minimum to about $100 \mathrm{~N}$ (Figure 6a). However, the left and right vastus medialis and lateralis muscles forces reached their initial maximum prior to the foot contact and their global minimum right after the foot contact (Figure 6b). After the foot contact, muscle forces increased rapidly to their maximum of about $780 \mathrm{~N}$. Hip, knee and ankle joint angular curves during the movement are presented in Figures 7-9, respectively, with $0^{\circ}$ representing full extension of the hip and knee joint and $90^{\circ}$ of the ankle joint. 


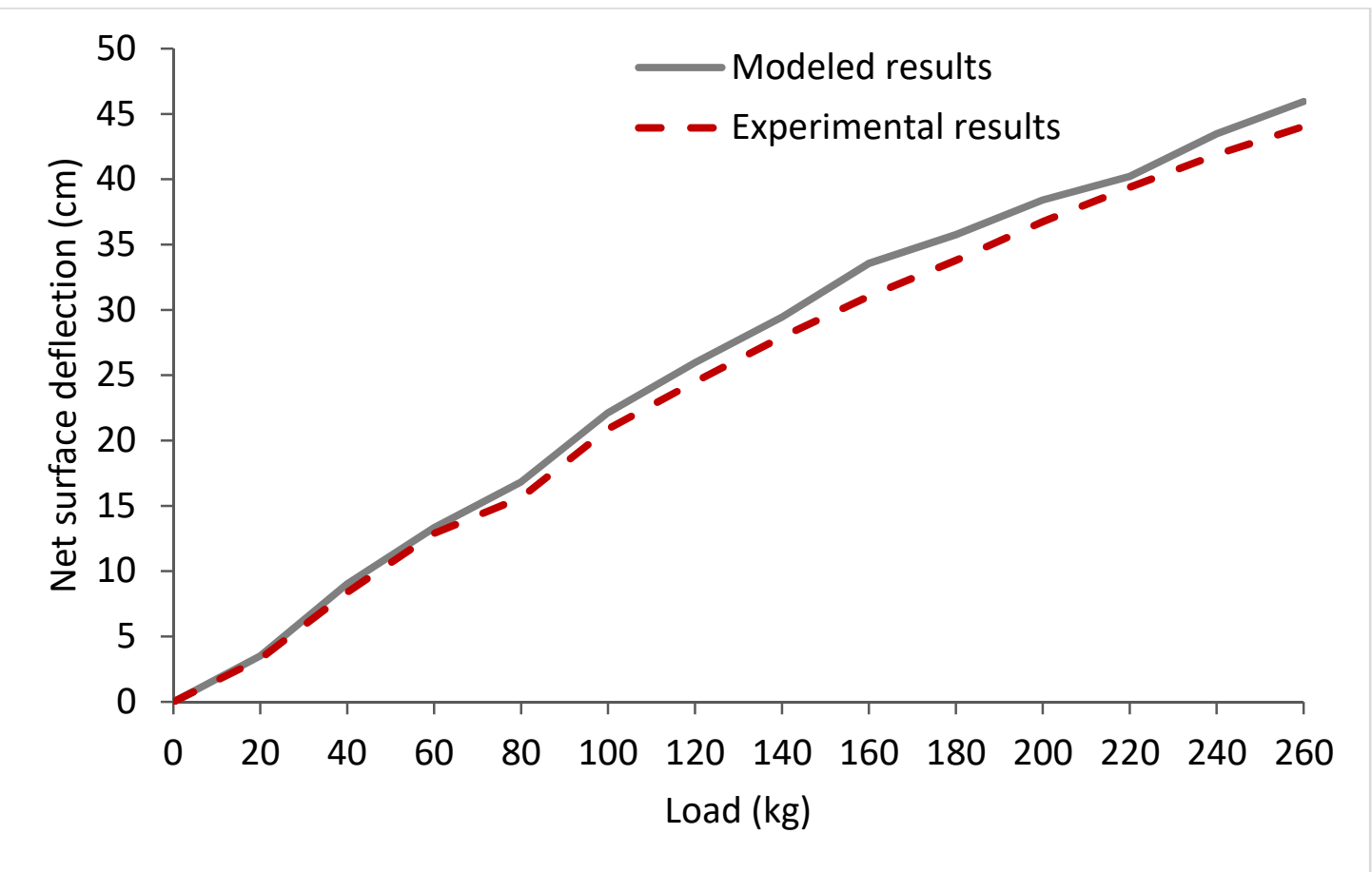

Figure 1

Comparison between modeled and experimental results in the static load tests.

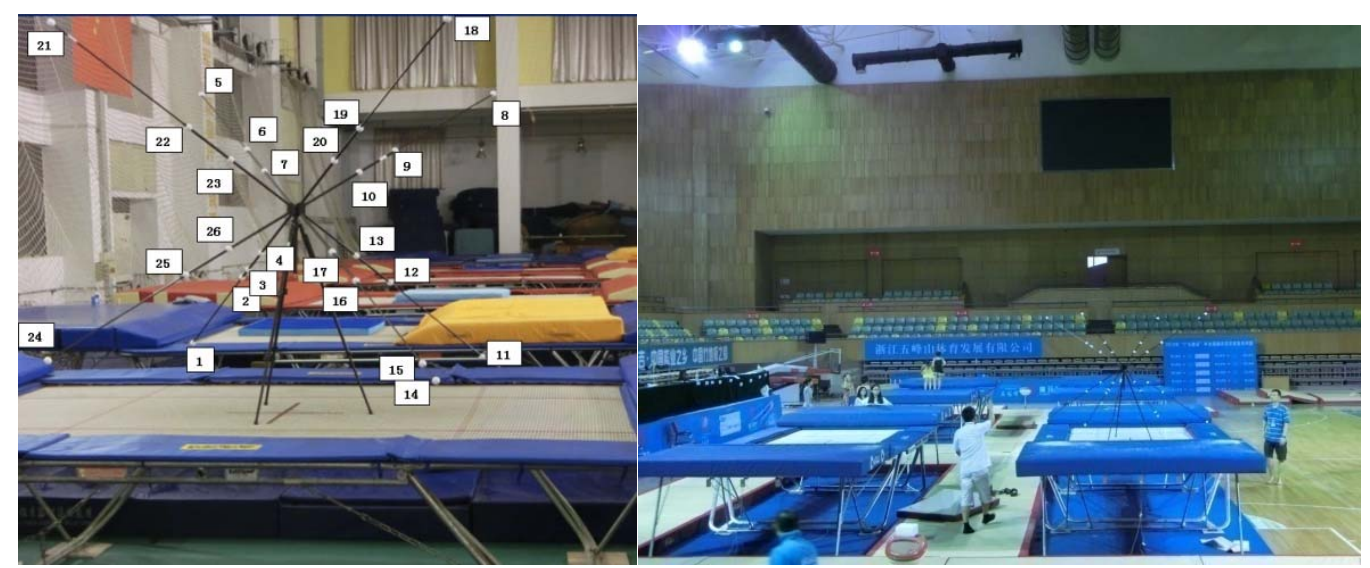

Figure 2

$3 D$ calibration frame and two high-speed digital video camcorders. 


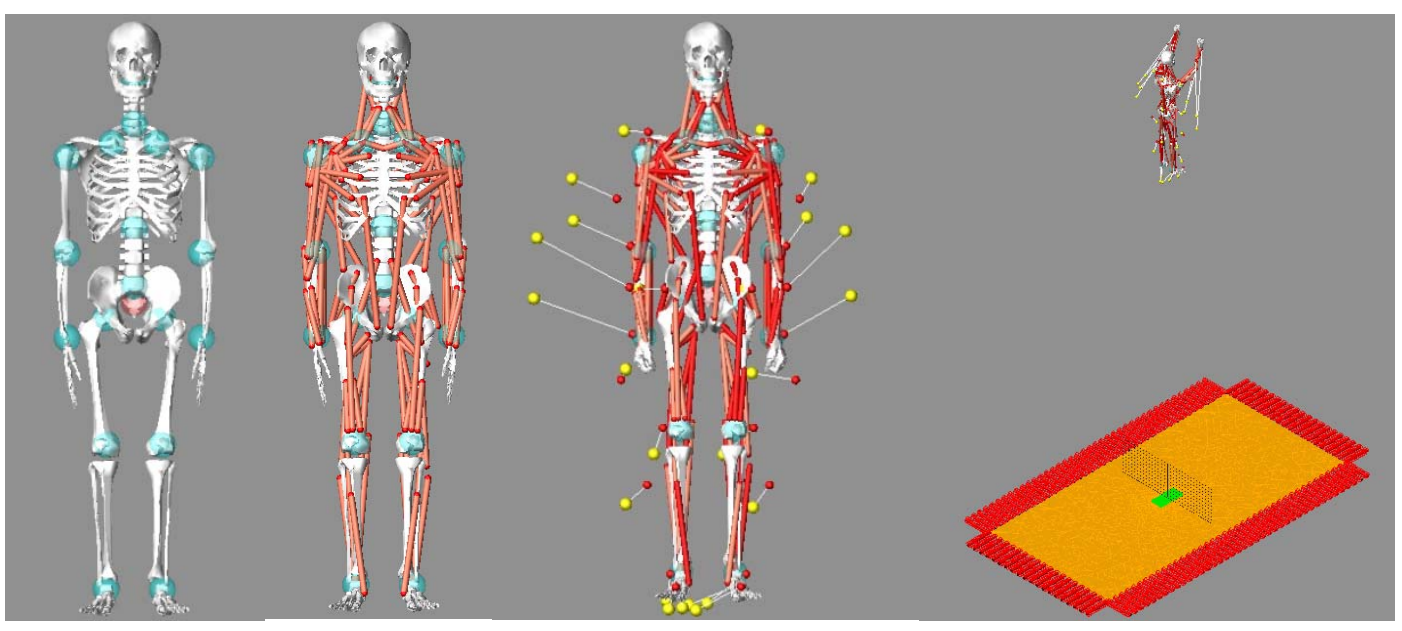

a

$\mathrm{b}$

$\mathrm{c}$

d

Figure 3

The musculoskeletal model (a) with muscles (b) and prescribed motions (c), and the finite element model of the trampoline combined with the musculoskeletal model (d).

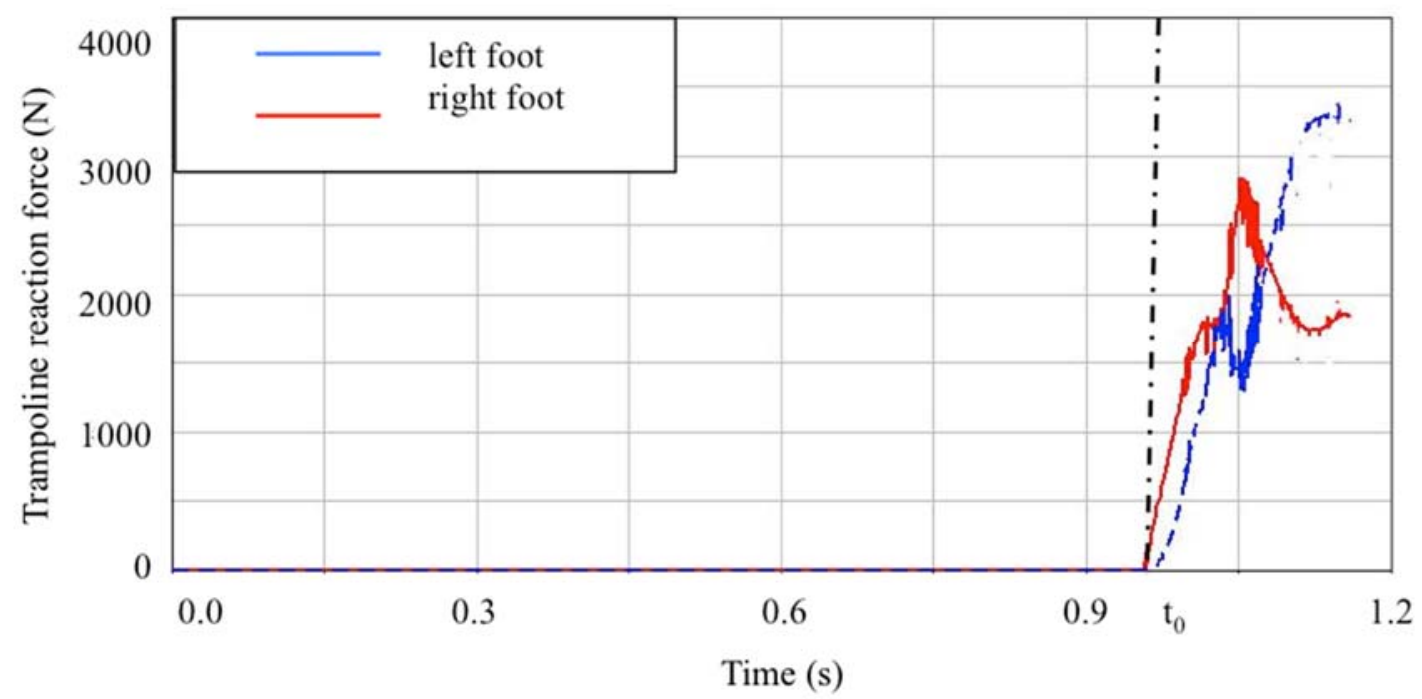

Figure 4

Trampoline reaction force. 


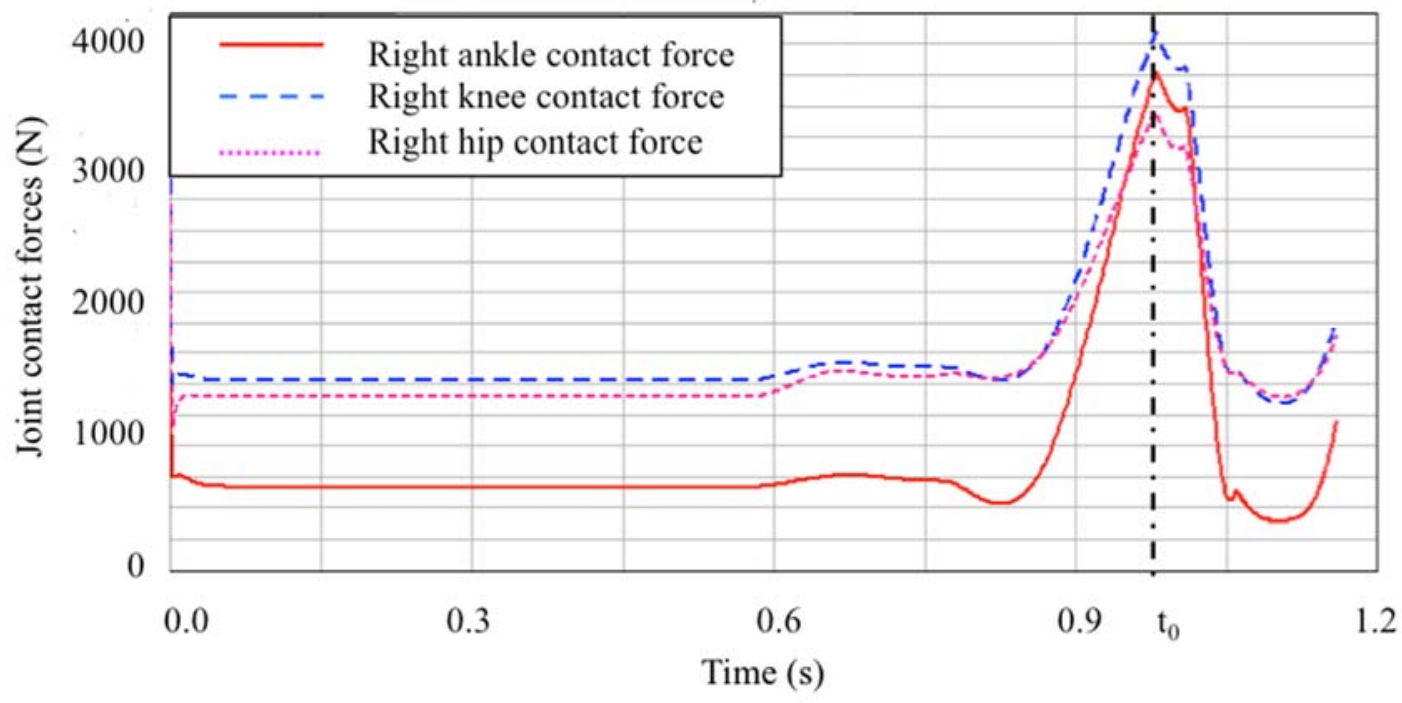

a)

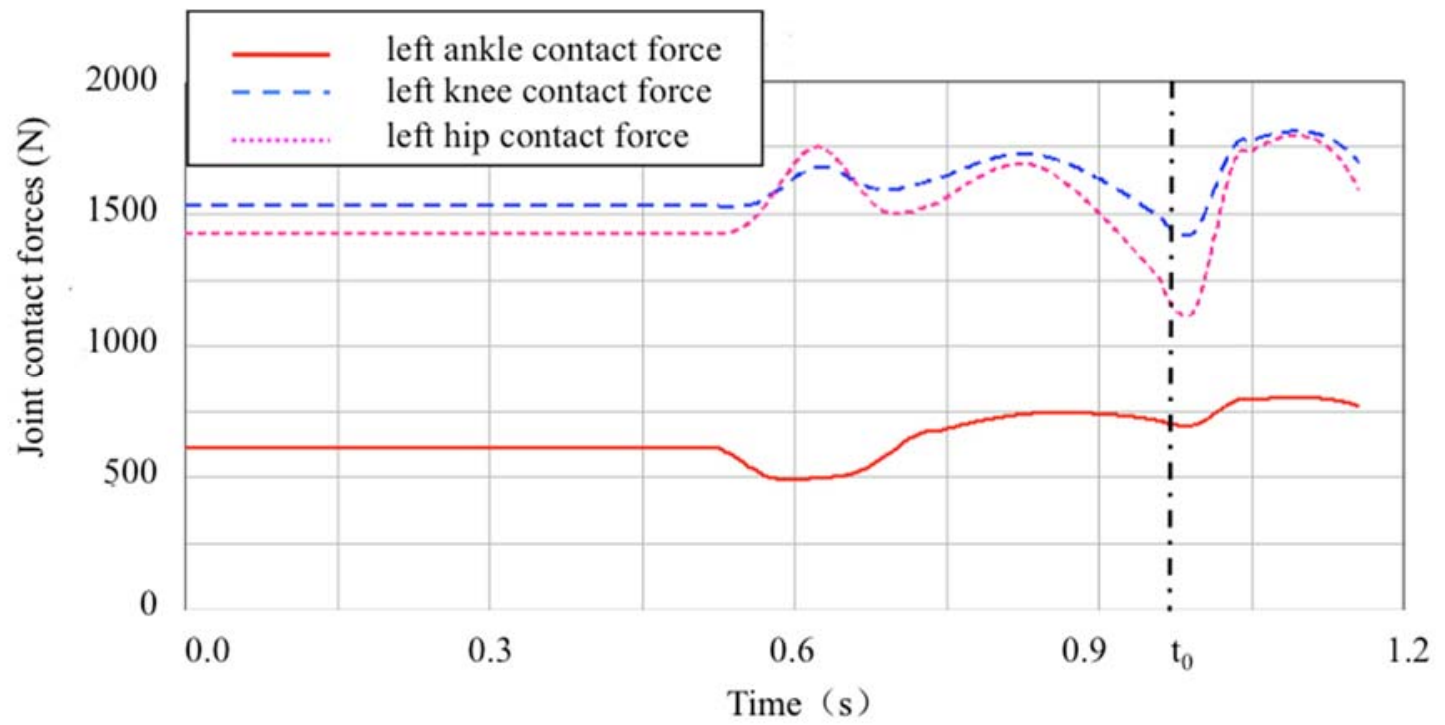

Figure 5

b)

Joint contact forces of a) right and b) left lower extremities. 


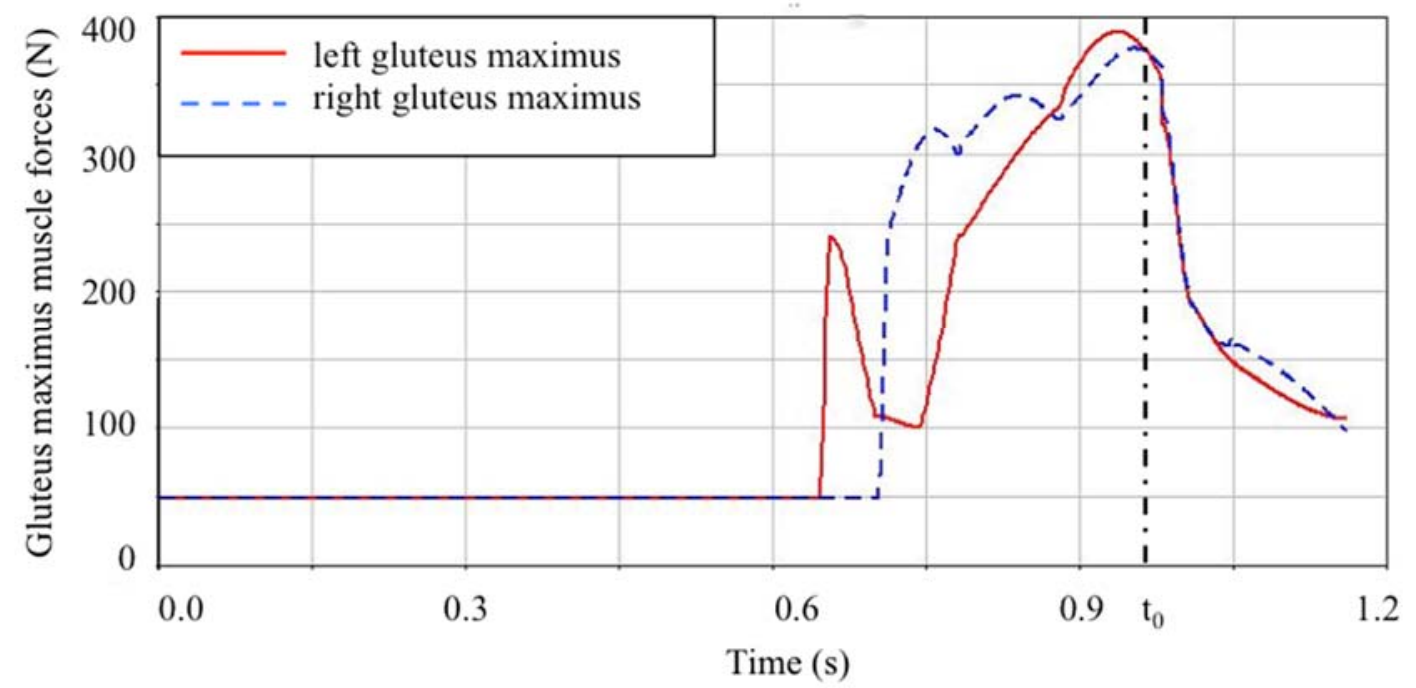

a)

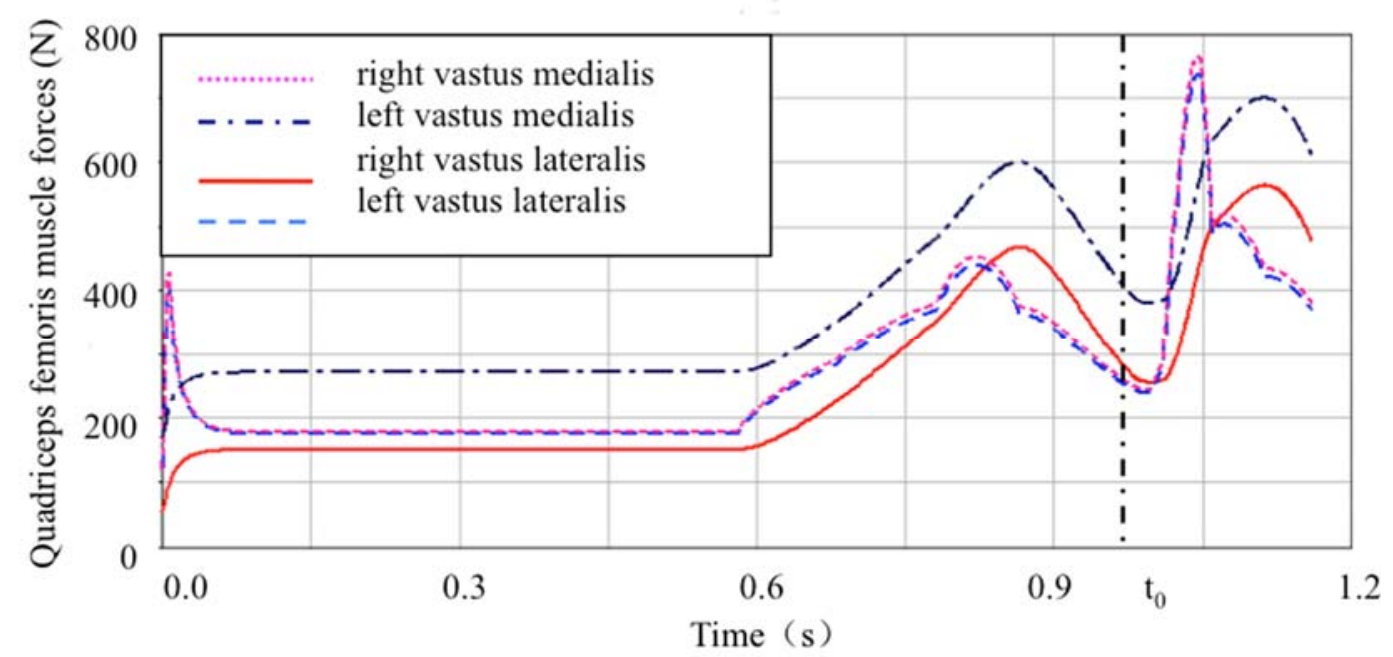

b)

Figure 6

a) gluteus maximus and b) vastus medialis and lateralis muscle forces. 


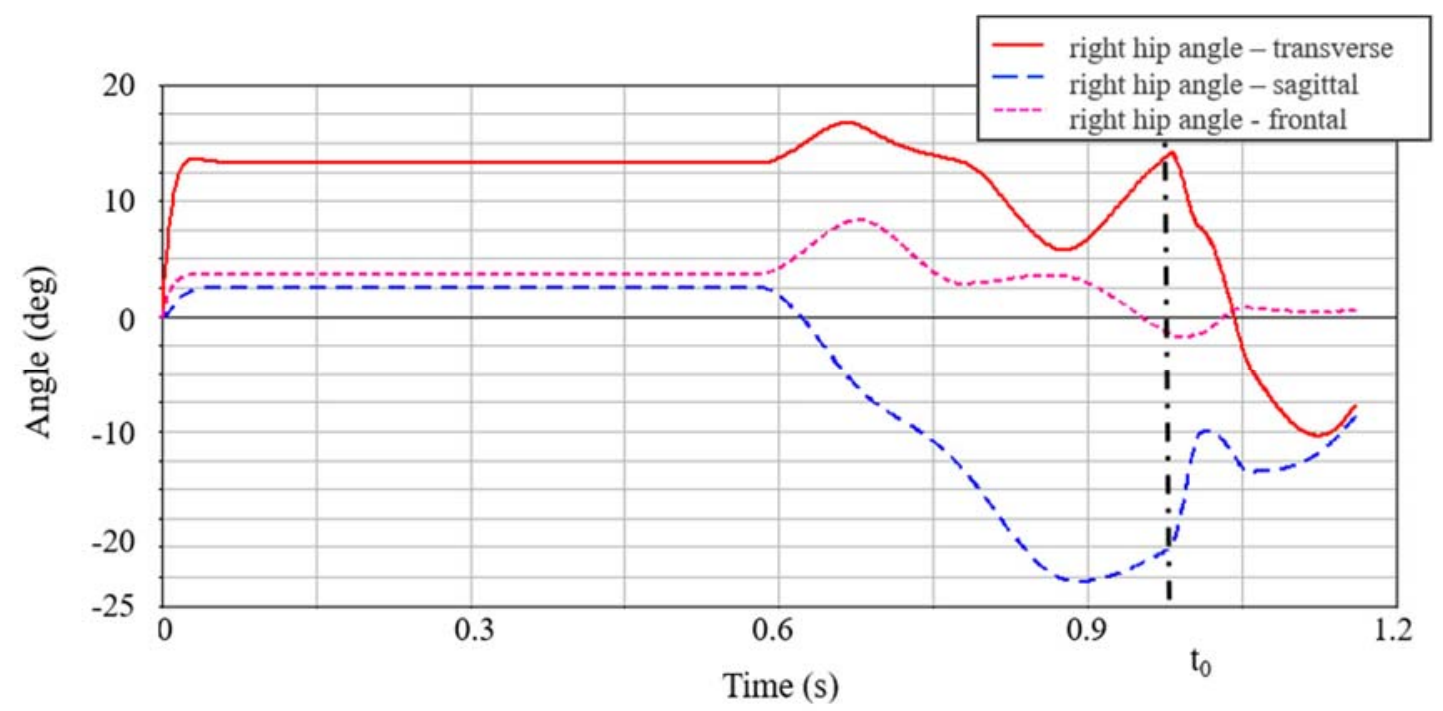

a)

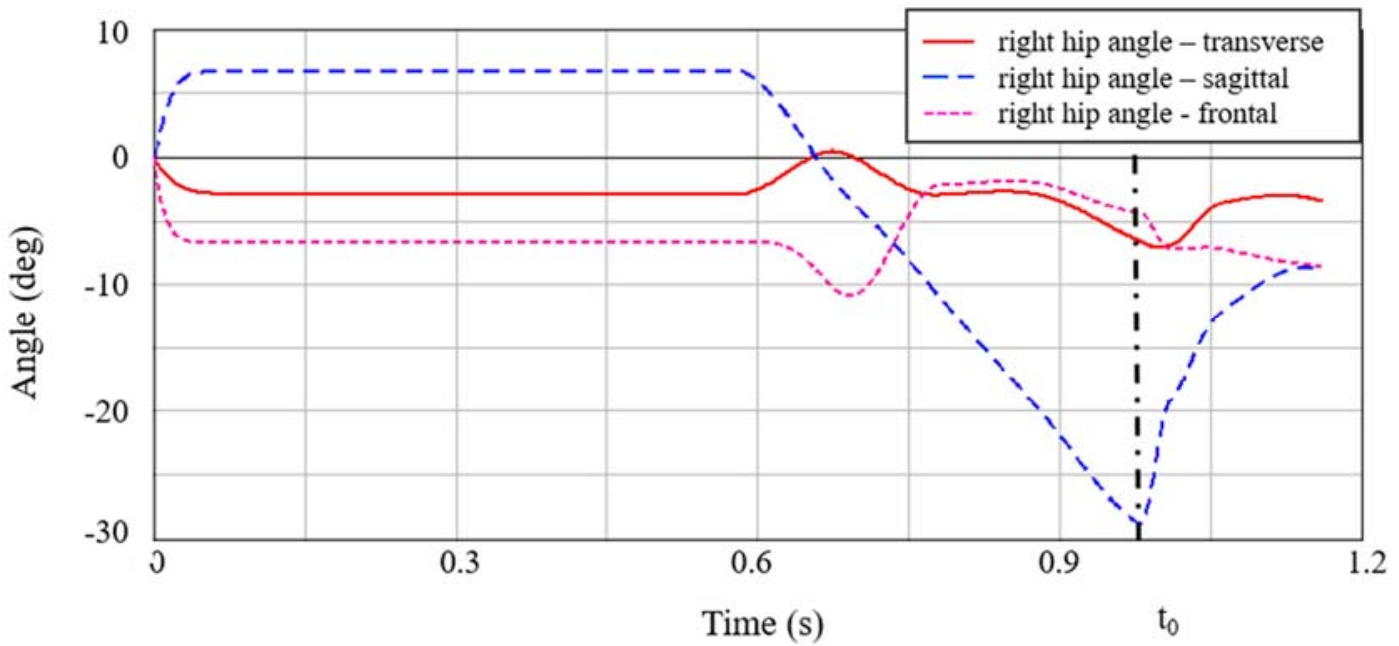

b)

Figure 7

Joint angle trajectory of a) right hip and b) left hip joint. 


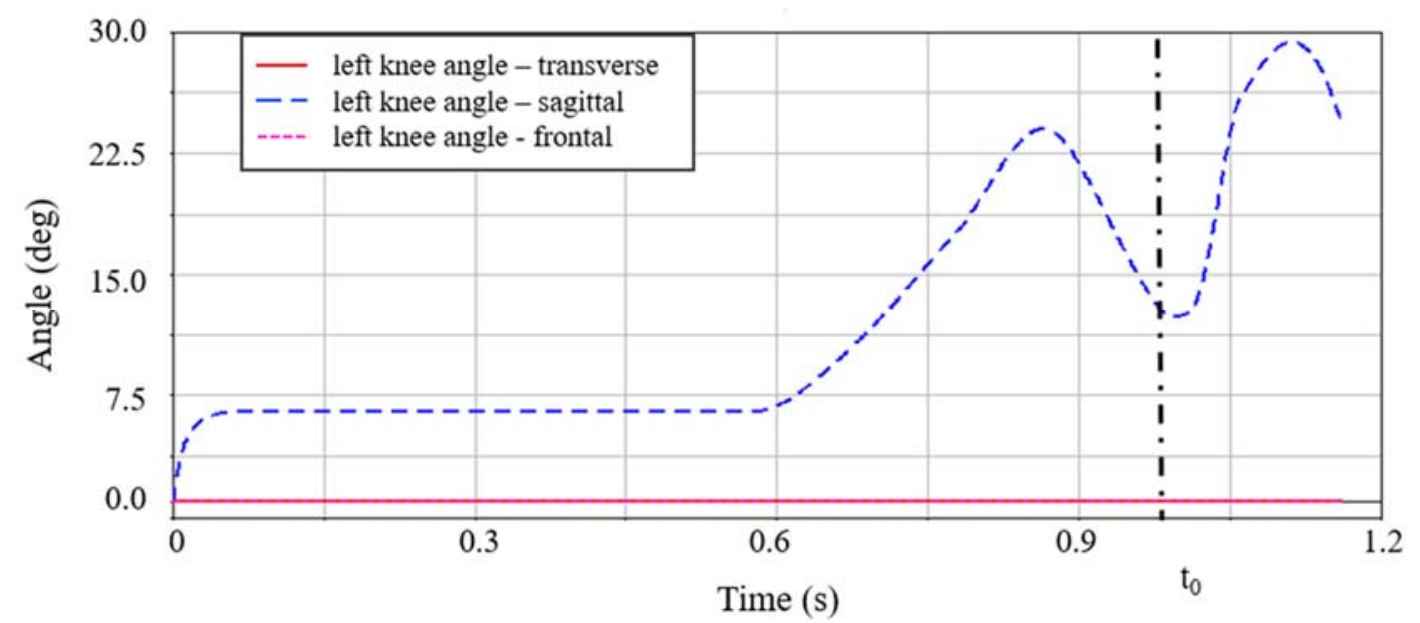

a)

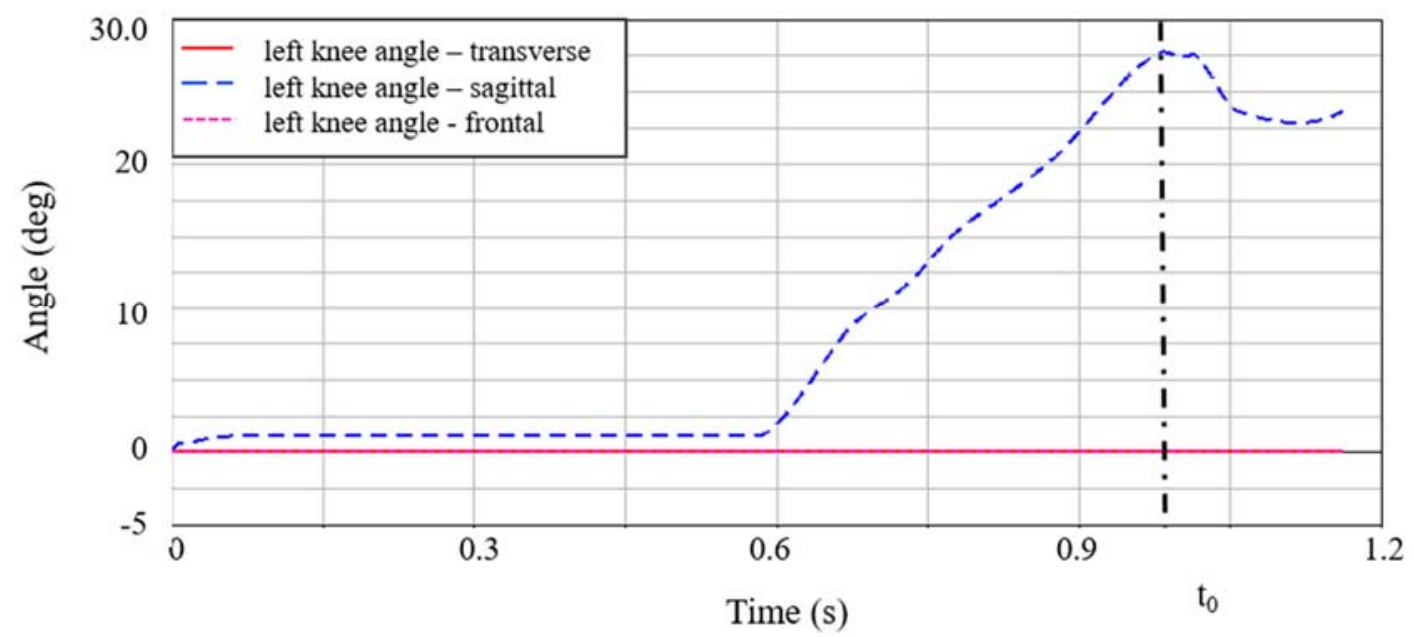

b)

Figure 8

Joint angle trajectory of a) the right knee and b) the left knee joint. 


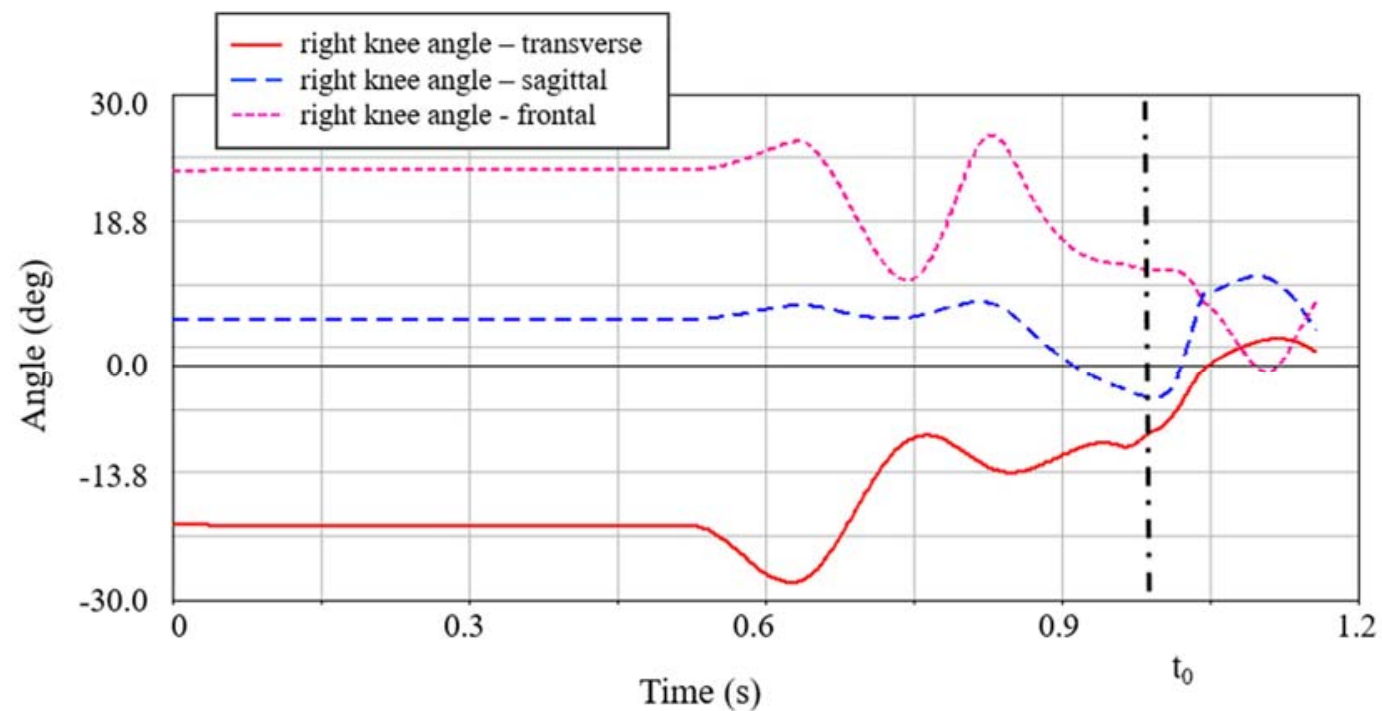

a)

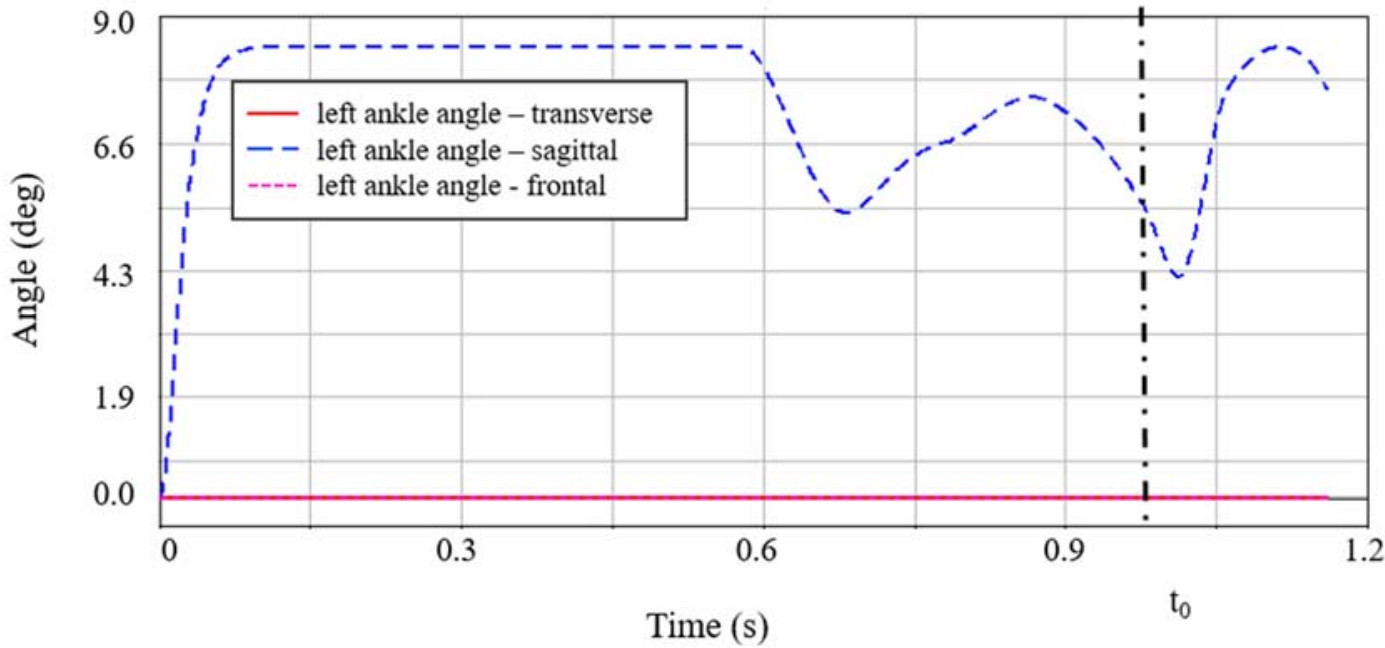

b)

Figure 9

Joint angle trajectory of a) the right ankle and $b$ ) the left ankle joint. 


\section{Discussion}

The main purpose of this study was to examine dynamics and selected lower extremity muscle forces in a landing and jumping movement on a trampoline, using a combination of finite element modeling and musculoskeletal modeling. During the descent phase, the athlete adjusted the posture and stiffened lower limb angles in order to transfer the kinetic energy to elastic energy of the trampoline net structure. At the same time, the hip and knee joints extended to reach their peak angles while ankle joints maintained their angles, in order to create a more rigid body posture to minimize energy loss during the net contact/takeoff process, prior to the foot contact (Figures 7 - 9). However, the right knee flexion angle was seen to increase slightly after the contact. The estimated peak TRFs reached 6.6 $\mathrm{BW}$ and 5.6 $\mathrm{BW}$ for the left and right limb, respectively, as the athlete was dropped from a very high height and landed onto the rather compliant net surface. Those force magnitudes are similar to the forces estimated from kinematics during different trampoline exercises (Vaughan, 1980). The lower limb posture prior to, at, and soon after the initial net contact is necessary to allow the athlete to interact with the deformation of the net surface during the body descent after the contact in order to attenuate impact forces, maximize jump height, and achieve a successful jump. Furthermore, the TRF reached different peak values and at different times after the net contact for the left and right limb (Figure 4), indicating that some degrees of asymmetry between left and right lower limbs may generate rotational motion, which is needed to perform subsequent movement once the jump is completed. These are supported by the asymmetrical angular motions of the left and right ankles and knee joints. Figure 9 showed that the right ankle experienced relatedly large frontal- and transverse-plane motions, while the left ankle had no observed changes in motion in these two planes (Figure 9a and 9b). Furthermore, changes in the right knee sagittal-plane motion were slightly smaller than those of the left knee (Figure 8a and $8 b)$.

In the current study, joint reaction forces of the right hip, knee and ankle joints reached their maximum between 5.8 and $7.7 \mathrm{BW}(3000-4000 \mathrm{~N})$ at the foot contact with the trampoline. After the net contact during the body descent, the peak TRF reached 6.6 BW $(3400 \mathrm{~N})$ and 5.6 BW $(2900 \mathrm{~N})$ for the left and right limb, respectively. The high compliance of the trampoline net contributes to the reduced peak TRF and joint reaction forces during the landing on the trampoline compared to the peak joint reaction forces of $16.6 \mathrm{BW}$ for the ankle, 11.9 BW for the knee and 12.2 BW for the hip in the landing on the less compliant mat during a vault exercise (Mills et al., 2009). A previous trampoline simulation study $(\mathrm{Lv}, 2012)$ showed that the peak TRF reached 7.9 BW (3500 N) after the net contact, and the peak hip, knee and ankle reaction forces reached 4.9 BW $(2160 \mathrm{~N}), 7.1 \mathrm{BW}(3132 \mathrm{~N})$ and 7.8 BW (3443 N), respectively, during the landing phase, which were similar to our findings. However, the model was based on a multi-segment rigid-body model and did not use a musculoskeletal modeling approach as employed in this study.

Both left and right gluteus maximus reached the maximum muscle force of $0.73 \mathrm{BW}$ $(380 \mathrm{~N})$ around the foot contact, while left and right quadriceps muscle reached the maximum values of $1.5 \mathrm{BW}(780 \mathrm{~N})$ after the foot contact at about the same time. They were the major muscle groups used during the trampoline-landing phase. Our muscle force results were partially supported by the findings in the literature. $\mathrm{Li}$ (2012) reported the gluteus maximum reached the peak muscle force about $1.8 \mathrm{BW}(800 \mathrm{~N})$ right before the net contact which was slightly higher than our results, whereas vastus medialis and lateralis were not involved in the net contact. Mills et al. (2009) demonstrated that the peak hip extensor and knee extensor muscle forces were 11.4 BW (8388 N) and $4.9 \mathrm{BW}(3580 \mathrm{~N})$ when landing on a gymnastic mat in a vault exercise, which were much higher than our results due to the lower shock absorption capability of the more rigid landing surface.

It is interesting to note the joint kinematics of this study is somewhat counter-intuitive. We initially anticipated that the athlete would flex the lower limb joints after the net contact in order to absorb impact. This contact movement would depress the net surface, and the athlete would then extend lower limb joints to depress the net to its lowest point and complete the takeoff with the aid of elastic recoil of the trampoline net surface. However, the actual joint kinematics of the athlete's performance showed the lack of the absorption movements of lower limb joints during the takeoff movement on the trampoline. This is 
similar to the technique we observed and summarized previously, which showed a rigid lower limb posture during "net entry" and a rapid explosive extension of the lower limb during the takeoff movement on a trampoline (Chen et al., 2014). These movement characteristics are partially supported by the simulation results. To our knowledge, this is the first musculoskeletal simulation study of the trampoline takeoff movement in the literature. Future studies are warranted to examine and confirm if the joint kinematics and dynamics, and muscle forces found in this study were unique to this athlete and the movement or are more universal.

\section{Conclusions}

This study is the first attempt to integrate musculoskeletal modeling with finite element modeling in examining dynamic interactions of the human body and a trampoline during a trampoline jump movement. The participant contacted the trampoline net with a relatively stiff body posture prior to the initial contact. Asymmetrical loading patterns between left and right TRFs and lower extremity joint reaction forces were evident due to the need to generate the rotational movement during the takeoff. The results also demonstrated that the gluteus maximus and quadriceps had different force patterns and played important roles in energy absorption and generation, and were responsible for asymmetrical loading and generation of rotation after the takeoff. Future research should focus on the causal relationship between lower muscle activations and movement control strategies of complex trampoline exercises with rotational components.

\section{Acknowledgements}

The work was supported by a grant from the Natural science foundation of Jiangsu province (Project number BK201288) and a grant from the National Natural Science Foundation of China (No. 31270998).

\section{References}

Ando T, Yamamoto H. Biomechanical Analysis of Trampoline Exercise. The 5th International Symposium on Biomechanics in Sports, 1987

Arora V, Kimmel LA, Yu K, Gabbe BJ, Liew SM, Kamali Moaveni A. Trampoline related injuries in adults. Injury, 2016; 47: 192-196

Atilgan OE. Effects of trampoline training on jump, leg strength, static and dynamic balance of boys. Science of Gymnastics Journal, 2013; 5: 15-25

Blajer W, Czaplicki A. Modeling and inverse simulation of somersaults on the trampoline. Journal of Biomechanics, 2001; 34: 1619-1629

Blajer W, Czaplicki A. Contact Modeling and Identification of Planar Somersaults on the Trampoline. Multibody System Dynamics, 2003; 10: 289-312

Burke D, Yeadon F, Hiley M. Optimum technique for maximising forward somersault rotation in trampolining. 33rd International Conference of Biomechanics in Sports. Poitiers, France, International Society of Sport Biomechanics; 2015

Chen M, Wang T, Qian J. Analyses of takeoff movement characteristics on trampoline of Chinese elite trampoline athletes. Journal of Nanjing Institute of Sport (Natural Science), 2014; 13: 44-48

Eager D, Chapman C, Bondoc K. Characterisation of trampoline bounce using acceleration. Proceedings: the 7 th Australasian Congress on Applied Mechanics (ACAM 7), 9-12 December 2012, the University of Adelaide, North Terrace Campus/National Committee on Applied Mechanics of Engineers Australia. Engineers Australia; 2012

FIdG. Technical Regulations (Federation Internationale de Gymnastique). 2015

Khorashad RS. Experimental Analysis for Determination of Effective Mass of the Trampoline Suspension System Clinical and Experimental Medical Sciences, 2013; 1: 131-140 
Kuisis S, Camacho T, Dafel S, van Rensburg DJ. Kinetic profile and incidence of injuries among high performance trampoline gymnasts. ISBS-Conference Proceedings Archive; 2011

Li G. Biomechanical study of trampoline athletes Zhou Xinyi online take-off. Nanjing Sport Institute; 2012

Liu B. The Experimental Study on the Mechanical Properties of the Gymnastic Trampoline Net Surface and the Mathematical Modeling Analysis of It. [Thesis]: Taiyuan University of Technology; 2014

Lv C. Research and Simulation on dynamics of Trampolining. [Thesis]: Southeast University; 2012

Mills C, Pain MT, Yeadon MR. Reducing ground reaction forces in gymnastics' landings may increase internal loading. J Biomech, 2009; 42: 671-678

Peng Y, Zhu M, Tang X. Analysis of the Grey Correlation Degree of World Elite Male Trampoline Athletes -Concurrently on Dialectical Development between the Influencing Factors. Journal of Nanjing Institute of Physical Education (Natural Science), 2013; 12: 43-46

Qian J-G, Ye Q. A Finite Element Modeling for Performance Optimization in Elite Trampoline. Journal of Computational and Theoretical Nanoscience, 2016; 13: 6446-6450

Vaughan CL. A kinetic analysis of basic trampoline stunts. Journal of Human Movement Studies, 1980; 6: 236-251

Wang Z. Digital 3D Trampoline Simulating System: VHTrampoline. Chinese Journal of Computers, 2007; 30: 498504

\section{Corresponding author:}

\section{Songning Zhang, PhD and FACSM}

Biomechanics/Sports Medicine Lab

Department of Kinesiology, Recreation, \& Sport Studies

The University of Tennessee

1914 Andy Holt Avenue

Knoxville, TN 37996-2700

USA

Email: szhang@utk.edu 